\title{
Real Space Bogoliubov-de Gennes Equations Study of the Boson-Fermion Model
}

\author{
J. Krzyszczak, T. Domański And K.I. Wysokiński \\ Institute of Physics and Nanotechnology Centre \\ M. Curie-Skłodowska University \\ pl. M. Curie-Skłodowskiej 1, 20-031 Lublin, Poland
}

The boson-fermion model was proposed to describe superconductivity in short coherence length superconductors. In this work we use it to study impurity induced inhomogeneities in high temperature superconductors as found in numerous scanning tunnelling measurements. The model was formulated in real space and solved with help of Bogoliubov-de Gennes approach. Disorder in the boson or fermion subsystem is directly coupled to the superconducting order parameter and leads to severe changes of superconducting properties like local order parameter and density of states. We present the results for many impurities randomly distributed over otherwise clean and periodic two-dimensional square lattice.

PACS numbers: 71.10.Fd, 74.20.-z, 74.81.-g

\section{Introduction}

The boson-fermion (BF) model assumes the existence in the system of two kinds of interacting quantum objects [1] consisting of (usually) local and immobile bosonic particles (doubly charged preformed pairs) interacting with mobile fermions (electrons). It is the boson-fermion scattering which induces the condensation transition in bosonic and superconducting transition in fermionic subsystem [2]. In real materials, as e.g. doped high-temperature superconductors (HTS), one expects both bosonic and fermionic parameters to depend on the local environment and thus to be random variables.

Here we present results of calculations of such important parameters as local value of the gap parameter $\Delta_{i}$ and local density of states $N_{i}(E)$ at lattice site $i . \quad \Delta_{i}$ and $N_{i}(E)$ are subject to measurements with help of scanning tunnelling microscope (STM). The STM spectra of many high temperature superconductors [3], e.g. $\mathrm{Bi}_{2} \mathrm{Sr}_{2} \mathrm{CaCu}_{2} \mathrm{O}_{8+\delta}$, indeed show the intrinsic inhomogeneities of $\Delta_{i}$ and $N_{i}(E)$ extending over the scales of few nanometres. 
Even though the microscopic origin of these inhomogeneities remains unclear, some experiments show positive correlation between location of the oxygen dopant and the value of local order parameters. Based on this scenario theoretical approaches have been proposed in which authors calculate local properties of the system by means of real space Bogoliubov-de Gennes approach, using Hubbard [4], $t-J$ [5] or similar models with disorder in normal (density) or anomalous (Cooper) channel [6]. To account for possible sources of inhomogeneities we use the boson-fermion model [1] in the form

$$
\begin{aligned}
\hat{H}^{\mathrm{BF}} & =\sum_{i, j, \sigma} t_{i j} \hat{c}_{i \sigma}^{\dagger} \hat{c}_{j \sigma}+\sum_{i \sigma}\left(V_{i}^{\mathrm{imp}}-\mu\right) \hat{c}_{i \sigma}^{\dagger} \hat{c}_{i \sigma} \\
& +\sum_{i}\left(E^{\mathrm{B}}+\delta E_{i}^{\mathrm{B}}-2 \mu\right) \hat{b}_{i}^{\dagger} \hat{b}_{i}+\sum_{i, j} \frac{g_{i j}}{2}\left(\hat{b}_{i}^{\dagger} \hat{c}_{i \downarrow} \hat{c}_{j \uparrow}+\hat{b}_{i} \hat{c}_{i \uparrow}^{\dagger} \hat{c}_{j \downarrow}^{\dagger}\right),
\end{aligned}
$$

where $i$ and $j$ denote lattice sites of the square lattice, $\hat{c}_{i, \sigma}^{\dagger}\left(\hat{c}_{i, \sigma}\right)$ stand for creation (annihilation) operator of fermion at the site $i$ with spin $\sigma$ and $\hat{b}_{i}^{\dagger}$ and $\hat{b}_{i}$ are creation and annihilation operators of hard-core bosons at the site $i$. $\mu$ denotes chemical potential of the system and $t_{i j}$ are hopping integrals. For numerical calculations we assume hopping integrals to nearest and next nearest neighbours $t_{1}, t_{2}$ to be different from zero. As noted earlier, the presence of oxygen impurities in Bi family of HTS on one hand is the source of free carriers (holes) and at the same time introduces disorder into the materials. Undoped system is insulating. We assume that this disorder can be modelled by random on-site energies $V_{i}^{\text {imp }}$ in fermion subsystem and random hard-core boson energies $\delta E_{i}^{\mathrm{B}}[7,8]$. To account for the short coherence length and $d$-wave symmetry of the superconducting order parameter we assume that the boson-fermion coupling $g_{i j}$ takes on non-zero value for nearest neighbour sites $\langle i, j\rangle$ only and is equal $+g$ if $j=i \pm \boldsymbol{x}$ and $-g$ if $j=i \pm \boldsymbol{y}$.

Application of standard Hartree-Fock-Bogoliubov decoupling leads to $\hat{H}^{\mathrm{BF}}=\hat{H}^{\mathrm{B}}+\hat{H}^{\mathrm{F}}$, where $\hat{H}^{\mathrm{B}}$ is single site bosonic Hamiltonian with parameters depending on the fermionic order parameter. $\hat{H}^{\mathrm{F}}$ is the Hamiltonian describing fermionic subsystem [8]. Standard statistical approach allows the exact solution of the boson Hamiltonian $\hat{H}^{\mathrm{B}}$. One finds

$$
\begin{aligned}
& \left\langle\hat{b}_{i}^{\dagger} \hat{b}_{i}\right\rangle=\frac{1}{2}-\frac{E^{\mathrm{B}}+\delta E_{i}^{\mathrm{B}}-2 \mu}{4 \gamma_{i}} \tanh \left(\frac{\gamma_{i}}{k_{\mathrm{B}} T}\right), \\
& \left\langle\hat{b}_{i}\right\rangle=-\frac{\chi_{i}}{2 \gamma_{i}} \tanh \left(\frac{\gamma_{i}}{k_{\mathrm{B}} T}\right) .
\end{aligned}
$$

Here

$$
\gamma_{i}=\sqrt{\left(\frac{E^{\mathrm{B}}+\delta E_{i}^{\mathrm{B}}-2 \mu}{2}\right)^{2}+\left|\chi_{i}\right|^{2}}, \quad \chi_{i}=\sum_{\langle j\rangle} \frac{g_{i j}}{2}\left\langle\hat{c}_{i, \downarrow} \hat{c}_{j, \uparrow}\right\rangle
$$

and $\Delta_{i j}=\frac{g_{j i}}{2}\left\langle\hat{b}_{j}\right\rangle$. It is $\Delta_{i j}$ which couples two subsystems as is evident from Eq. (4) below. 
The fermion part has the standard BCS-structure and we diagonalise it by the Bogoliubov-Valatin transformation, which yields the following Bogoliubov-de Gennes equations [8]

$$
\sum_{j}\left(\begin{array}{c}
t_{i j}+\left(V_{i}^{\mathrm{imp}}-\mu\right) \delta_{i j} ; \Delta_{i j} \\
\Delta_{i j}^{*} ;-t_{i j}-\left(V_{i}^{\mathrm{imp}}-\mu\right) \delta_{i j}
\end{array}\right)\left(\begin{array}{c}
u_{j}^{l} \\
v_{j}^{l}
\end{array}\right)=E^{l}\left(\begin{array}{c}
u_{i}^{l} \\
v_{i}^{l}
\end{array}\right) .
$$

We solve numerically the above Bogoliubov-de Gennes equations in real space iteratively to get self-consistent values for all $\Delta_{i j}$ with high accuracy. Even though our model and calculations are motivated by the experimental results obtained mainly for Bi family high temperature superconductors we do not try to model the actual experimental situation here. Instead we shall present the results on local properties of disordered boson-fermion model. In this respect the paper is an extension of the previous coherent potential approximation (CPA) calculations [8] of the same model.

\section{Results and discussion}

Before we start the discussion of the results let us remind that it is the position of the boson level with respect to the Fermi level $E_{\mathrm{B}}$ which tells us if the system is superconducting at all. Due to phase space restrictions at low temperatures the scattering of preformed bosons is allowed only, if the bosonic level is close enough to the Fermi level.

To account for disorder in the system the boson energies $E_{i}^{\mathrm{B}}=E^{\mathrm{B}}+\delta E_{i}^{\mathrm{B}}$ were randomly and uniformly distributed around level $E^{\mathrm{B}}$ over the interval $\Delta E^{\mathrm{B}}$. Physically this corresponds to fluctuating pairing potential [6], which in BF model arises in a very natural way.

Due to the above mentioned property of the model the magnitude of the local order parameter $\left\langle b_{i}\right\rangle$ (not shown) obtained as a solution of Eq. (4) is correlated with the position of the impurity provided we identify the dopant oxygens as e.g. those in $\mathrm{Bi}_{2} \mathrm{Sr}_{2} \mathrm{CaCu}_{2} \mathrm{O}_{8+\delta}$ with the pairing centres. From the same calculations it can also be seen that both order parameters $\left\langle b_{i}\right\rangle$ and $\chi_{i}$ take on large values in the same regions of space. The direct proportionality of both order parameters is a general property of the model [2], which can also be inferred from the mean-field Eq. (3).

In Fig. 1 we show the effect of disorder in bosonic (random $E_{i}^{\mathrm{B}}$ levels left part) and fermionic (random $V_{i}^{\mathrm{imp}}$ - right part) subsystems on the average values of the order parameters $\left|\left\langle b_{i}\right\rangle\right|$ and $\left|\chi_{i}\right|$. In this figure the clean system (characterised by $E_{\mathrm{B}}=-0.2 t$ ) would correspond to the experimental situation with all sites contributing to boson-fermion scattering. With increase in $\Delta E^{\mathrm{B}}$ the number of sites at which this scattering takes place diminishes and the order parameter is strongly reduced.

It is interesting to note that despite the $d$-wave character of superconductivity the disorder acting on the fermionic subsystem relatively weakly affects both 


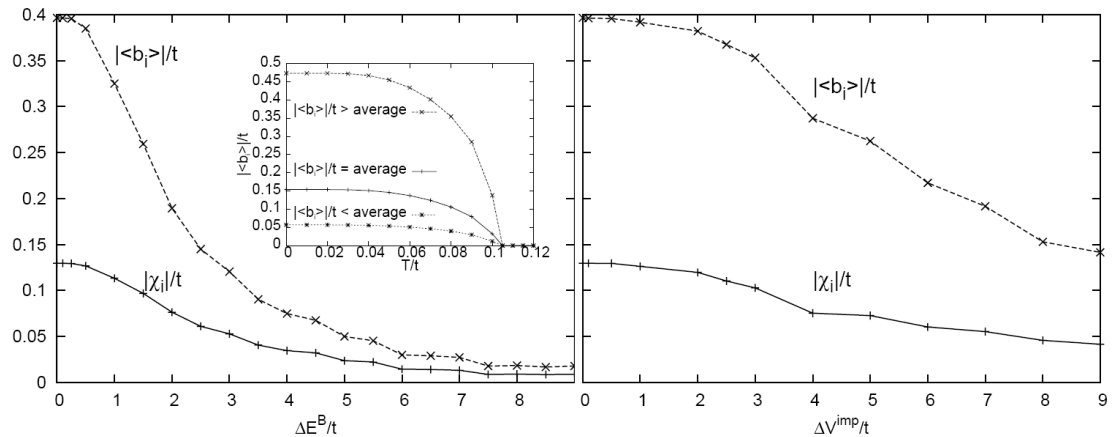

Fig. 1. Average values of $\left|\left\langle b_{i}\right\rangle\right|$ and $\left|\chi_{i}\right|$ as a function of disorder in the boson (left part) and fermion (right part) subsystem. Inset to left part: The temperature dependence of an average $\left|\left\langle b_{i}\right\rangle\right|$ compared with similar dependence at the sites at which the value of the gap is larger/smaller than the average. The results were obtained for $t_{1}=t=1.0$, $t_{2}=-0.3 t, \mu=0.0 t, g=0.8 t, E_{\mathrm{B}}=-0.2 t$.

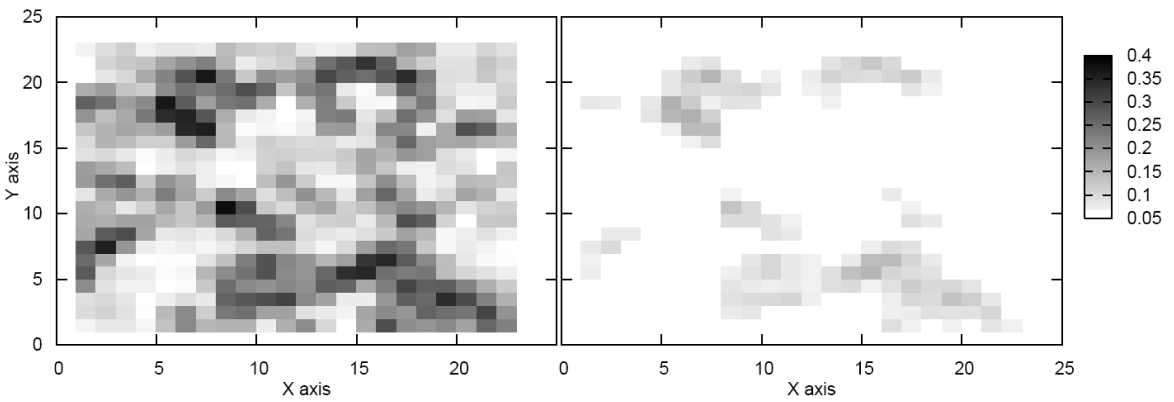

Fig. 2. Maps of amplitude of bosonic order parameter $\left|\left\langle b_{i}\right\rangle\right|$ for different values of temperature: $T=0.0 t$ (left part), $T=0.1 t \approx 0.9 T_{\mathrm{c}}$ (right part). Other parameters are the same as in Fig. 1.

order parameters (Fig. 1, right part). More severe changes of both $\left|\left\langle b_{i}\right\rangle\right|$ and $\left|\chi_{i}\right|$ are observed for very large disorder. The results presented in inset to Fig. 1 (left) show that the whole system is characterised by the unique transition temperature. This result is correct from thermodynamical point of view. At the same time it indicates that for the system studied here there exists a coupling between regions of large and small pairing potential, preventing the appearance of isolated regions with different values of the transition temperatures, even though the gaps near $T_{\mathrm{c}}$ do vary a lot as is also visible from Fig. 2 - right part. Indeed, rough estimation of the coherence length leads to values of order $1 / 3$ to $1 / 2$ of the system size. This shows that all domains are coupled. In Fig. 2 we plot the maps of bosonic order parameter $\left|\left\langle b_{i}\right\rangle\right|$ of our $23 \times 23$ large system at different temperatures. They are shown in grey scale for two values of temperature $-T \ll T_{c}$ (left part) and for $T \approx 0.9 T_{\mathrm{c}}$ (right part). At temperatures close to $T_{\mathrm{c}}$ the regions of large gap seem 
to be surrounded by the non-superconducting material. Close inspection shows that the whole system is superconducting but most of it is characterised by very low value of the gap, while large gap persists in few regions of the sample. We do not address the pseudogap problem here, as our mean-field approach is not suitable in that region of parameter space.

In conclusion, we studied the properties of disordered boson-fermion model allowing for two different disorder sources. The randomness in boson energies strongly degrades superconductivity, while disorder acting on electron subsystem $V_{i}^{\text {imp }}$ has much weaker effect on superconducting state. The local values of fermionic order parameter positively correlate with positions of "pairing impurities" characterised by small values of $E_{\mathrm{B}}$. Even strongly disordered system possesses single superconducting transition temperature despite large fluctuations of the gap magnitude.

\section{Acknowledgments}

This work was partially supported by the grant no. N N202 187833.

\section{References}

[1] J. Ranninger, S. Robaszkiewicz, Physica B 135, 468 (1985).

[2] T. Kostyrko, J. Ranninger, Acta Phys. Pol. A 91, 399 (1997).

[3] K. McElroy, J. Lee, J.A. Slezak, D.H. Lee, H. Eisaki, S. Uchida, J.C. Davis, Science 309, 1048 (2005).

[4] T.S. Nunner, B.M. Andersen, A. Melikyan, P.J. Hirschfeld, Phys. Rev. Lett. 95, 17703 (2005).

[5] M.M. Maśka, Ż. Śledź, K. Czajka, M. Mierzejewski, Phys. Rev. Lett. 99, 147006 (2007).

[6] B.M. Andersen, A. Melikyan, T.S. Nunner, P.J. Hirschfeld, Phys. Rev. B 74, 060501 (2006).

[7] S. Robaszkiewicz, G. Pawłowski, Mol. Phys. Rep. 34, 76 (2001).

[8] T. Domański, K.I. Wysokiński, Phys. Rev. B 66, 064517 (2002); T. Domański, J. Ranninger, K.I. Wysokiński, Acta Phys. Pol. B 34, 493 (2003). 